\title{
Design and development of a soft robotic gripper for manipulation in minimally invasive surgery: a proof of concept
}

\author{
Giovanni Rateni · Matteo Cianchetti • \\ Gastone Ciuti · Arianna Menciassi • \\ Cecilia Laschi
}

Received: 1 November 2014/Accepted: 7 August 2015/Published online: 20 August 2015

(C) The Author(s) 2015. This article is published with open access at Springerlink.com

\begin{abstract}
This paper proposes the use of soft materials for building robotic grippers for delicate and safe interactions. The work includes concept design, fabrication and first assessment and characterization of the proposed soft gripper, a novel robotic end-effector entirely made up of elastomeric material. As a significant case study, it has been specifically adapted as a grasping tool in Minimally Invasive Surgery, but its design has been conceived in such a way that its dimension can be easily scaled, to find application in all those fields where a safe interaction with fragile items or human co-workers is needed. Moreover, the process is flexible for including further features to enrich its behaviour.
\end{abstract}

This work has been partially supported by the European Commission with the STIFF-FLOP IP (287728) and the ROBOSOFT CA (619319).

G. Rateni · M. Cianchetti · G. Ciuti ( $₫)$.

A. Menciassi - C. Laschi

The BioRobotics Institute, Viale Rinaldo Piaggio, 34, Pontedera, PI, Italy

e-mail: g.ciuti@sssup.it

G. Rateni

e-mail: g.rateni@sssup.it

M. Cianchetti

e-mail: m.cianchetti@sssup.it

A. Menciassi

e-mail: a.menciassi@sssup.it

C. Laschi

e-mail: c.laschi@sssup.it
Keywords Soft robotics - Minimally invasive surgery $\cdot$ Grasping tools for surgery $\cdot$ Compliance . Under-actuation

\section{Introduction}

Over recent years, technological developments in robotics have led to novel and less invasive techniques for surgery. In this framework, open surgery is often replaced by less invasive techniques, such as laparoscopy, towards Minimally Invasive Surgery (MIS). MIS has brought substantial benefits to the patient, such as: (1) less traumatization, (2) less risk of infection, (3) shorter recovery time and (4) a better cosmetics. In laparoscopic surgery, a single large incision is replaced by multiple small incisions (from 1 to $15 \mathrm{~mm}$ in diameter) through which physicians introduce long instruments for performing the medical procedure [23]. Due to advantage in reducing the surgical trauma, postoperative pain and cosmetic problems, the laparoscopic approach has been adopted in several medical fields, such as urological, thoracic and pediatric surgery.

Although much work has been done to develop dexterous, multi-degree of freedom forceps and grippers, they are still inadequate to grasp, manipulate or push aside internal organs. Force feedback or touch sensation is limited in the currently available MIS tools, thus creating in most cases the potential for excessive force application during surgery and 
unintended tissue injury $[3,19,26]$. The risk of complications due to traumatization of soft tissues while trying to securely grip them is still an unresolved issue using conventional instruments, often characterized by sharp edges and no compliant properties.

Current research is focused on improvement of traditional tools, adding compliant constructive strategies or implementing force-feedback controlled forceps, to limit the force exerted and preventing damages on the tissues [9, 18, 21, 27]. Alternatively, a smart-designed instrument made up of intrinsically compliant materials would avoid the use of complex force-feedback control or at least would automatically avoid possible damages by limiting the maximum applicable force.

In addition to the robotic surgery field, there exist different previous inspiring examples on the use of soft grippers. Here some of them have been reported without the aim of being exhaustive. In 1991 Suzumori et al. [25] already developed a device based on pneumatic actuators which is able to manipulate relatively small objects. More recently, an entire hand with high dexterity and capabilities which resemble the functionality of a human hand has been developed by Deimel and Brock [7]. In this case, the power source is fluidic, which guarantees compliance and relatively high interaction forces. Using the same principles, a soft gripper inspired by the star fish has been developed by Ilievski et al. [10], where the fluidic source is used to close the "fingers" around the objects. The main limit on using this actuation technology is represented by the low capability of miniaturization without a dramatic decrease of mechanical performance. Electro Active Polymers (EAP) are also a viable way of approaching miniaturization, but ionic EAP actuation velocity does not meet the usual timescale needed for surgical operations (Ionic Polimer Metal Composite based fingers in [2]); on the other hand, the high electric fields necessary for the electric EAP (the Dielectric Elastomer Minimum Energy Structure in [1]) do not allow a straightforward use of these technologies. A comparison between the reviewed existing soft robotic grippers and the system proposed in this work is presented in Table 1. Shape Memory Alloys (SMA), with their outstanding downscaling properties and high power density, could be a good alternative, but the high temperature and current necessary for their activation still represent an unsolved issue for their use in the surgical field. Only a few number of pioneering works exist for soft instruments applied in surgery [6, $13,24]$, but no one has still ventured into the design of a totally soft gripping tool for surgery. The application of soft robotics in surgery still represents a challenge, especially for precision tasks, because of the lack of reliable modelling and control algorithms [14].

This study proposes a method to exploit specific soft robotics technologies in the surgical field. The idea at the base of this proposal is to study the feasibility of grasping soft tissues by using a soft instrument based on under-actuated mechanisms. The advantages are all related to the intrinsic compliant properties of the elastomeric material chosen to fabricate the tool, which would allow getting safely closer to soft tissues inside the unstructured workspace of the abdominal cavity, without the risk of damaging blood vessels or delicate organs during the manipulating procedures.

\section{Soft Claw Gripper design}

This section describes the design of the Soft Claw Gripper (SCG), a manipulator totally made up of silicone material, which exploits its intrinsic compliance as a point of strength to achieve shape match. An adequate shape match increases the contact surface in grasping without sensing and control integration. It also increases the robustness to uncertainties in finger control and position, and the model of the environment. The approach is inspired by the biological model of the octopus.

In the octopus arm, an active bending requires selectively contracting the longitudinal muscle fibers along one side of the arm, thus creating an asymmetrical longitudinal compressional force that shortens one side of the arm and thus causes bending [12, 15]. Inside an elastomer segment, a longitudinal and eccentric arrangement of sheathed wires along its length is assimilable to a muscle longitudinal fiber and the pulling of a cable produces the selective contraction of the side subject to the action of the same cable.

Matching shape of an object without a complex control is possible by implementing an under-actuated mechanism, i.e. a mechanical concept with less control inputs (active joints) than Degrees Of Freedom (DOFs). This mechanism allows for an adaptive closure on the surface of an object and the grasping 
Table 1 Comparison table between the reviewed soft robotic grippers and the proposed work

+ Figure of merit

\begin{tabular}{llllll}
\hline & FMA & RBO hand 2 & Star fish & CSO gripper & SCG \\
\hline Actuation & Pneumatic & Pneumatic & Pneumatic & EAP & Cable-driven \\
Fabrication & - & + & + & -- & ++ \\
Control & - & + & + & - & + \\
Precision & + & + & + & - & - \\
Scalability & - & - & - & ++ & ++ \\
References & {$[25]$} & {$[7]$} & {$[10]$} & {$[1]$} & \\
\hline
\end{tabular}

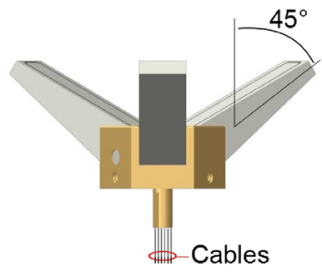

(a)

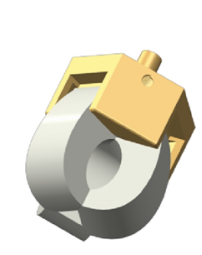

(c)

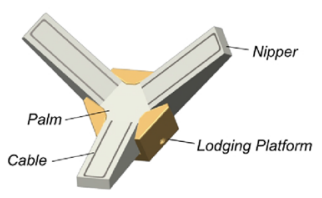

(b)

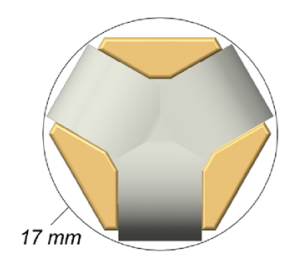

(d) interaction with the environment. A truncated pyramid shape was chosen, to meet a profile that forces the structure to bend itself in only one direction and to increase the stability during grasping (Fig. 2a, b).

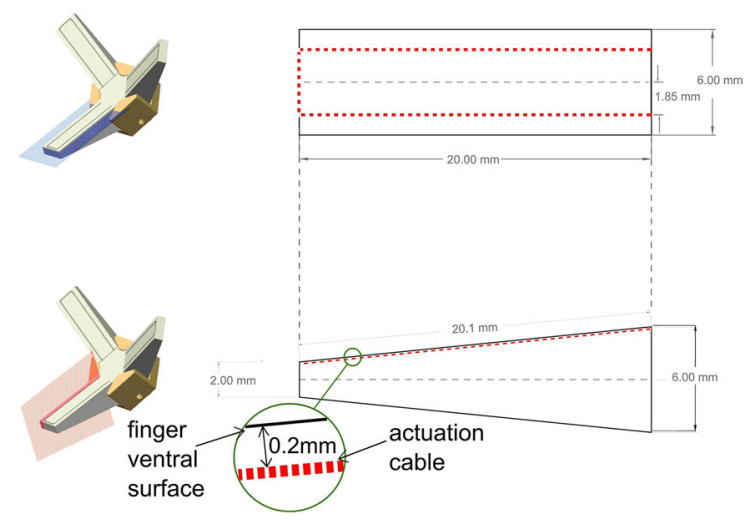

Fig. 1 Cut sections which show the positioning of the cable (dashed line) lying parallel to the ventral side of the finger. In particular, in a close-up detail, the distance of $0.2 \mathrm{~mm}$ between the finger ventral surface and the cable is shown. (Color figure online)

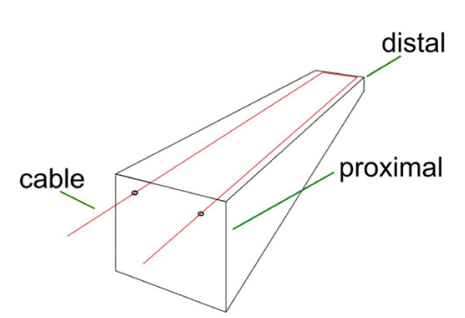

(e)

Fig. 2 Drawings of the Soft Claw Gripper: a front and $\mathbf{b}$ isometric view in open configuration; $\mathbf{c}$ isometric and $\mathbf{d}$ top view in closed configuration; e schematics of the actuator arrangement

Each finger of the SCG has infinite DOFs actuated by one cable and shows an under-actuated behaviour obtained combining the passive deformation property of the silicone and an innovative strategy of embedding sheathed cable inside. One nylon cable, previously inserted in a silicone sheath, is placed inside the finger with the central part fixed by two points at the tip and the rest running in two parts longitudinally 
down to its base, and coming out from the finger body. The plane containing the cable is parallel to the finger ventral surface and placed just below it (Fig. 2e). If a complex-shaped object is grasped, sliding along the sheath path, the gripper can passively adapt its surface to the object, while the cables tips are fixed to the actuation source (e.g. a servomotor). Then, two behaviours can be obtained simultaneously: (1) a curling motion on the sagittal mid-plane due to the pulling of the cable and (2) a 3-D shape matching motion due to the relative slide between gripper body and cable.

This work is devoted to analyze the possible application in the medical field, as a tool for manipulation of soft tissues in MIS, such as for single port laparoscopy (SPL) application [16]. To meet the constraints imposed by the single-port access diameter (maximum diameter of the skin incision in the abdomen of 30-35 $\mathrm{mm}$ [8]) and in particular to find an immediate translation in a robotic application for SPL (integration in the SPRINT robot with arms of 18 $\mathrm{mm}$ in diameter $[20,22])$, the total diameter of the system should not exceed $18 \mathrm{~mm}$. It is possible to insert the system in closed configuration, with a hindrance of $17 \mathrm{~mm}$ (Fig. 2c, d). The total length of each finger has been fixed at $20 \mathrm{~mm}$, representing it a reasonable length for a laparoscopic gripper. Each finger has a cross section of $6 \times 2 \mathrm{~mm}^{2}$ at the distal part and $6 \times 6 \mathrm{~mm}^{2}$ at the proximal part. The fingers are mounted so that they have an angle of $45^{\circ}$ between the vertical and the axis of lateral symmetry of the finger. In this specific case, the SCG has been designed to meet the aforementioned requirements for a SPL robotic procedure, but it can be easily designed to fit a standard trocar-based laparoscopic access port for standard tools $(<15 \mathrm{~mm}$ in diameter).

\section{Materials and methods}

\subsection{Fabrication phase}

The manufacturing process of the SCG is additive and uses 3D-printed molds. This makes the customization and combination of actuator shapes simple and supports the implementation of complex deformations. The gripper weighs $3 \mathrm{~g}$ and is obtained from a four-step casting procedure of a silicone in molds:
1. Three finger molds, 3 support bases and 1 lodging platform for each gripper are CAD-designed and 3D-printed (Fig. 3a). The molds are needed to cast the fingers into the desired shape with the cables properly placed in position. The support bases are used to ensure the correct positioning with the lodging platform during a second merging cast step;

2. Three molds are prepared with their respective actuation cables internally arranged. The cables

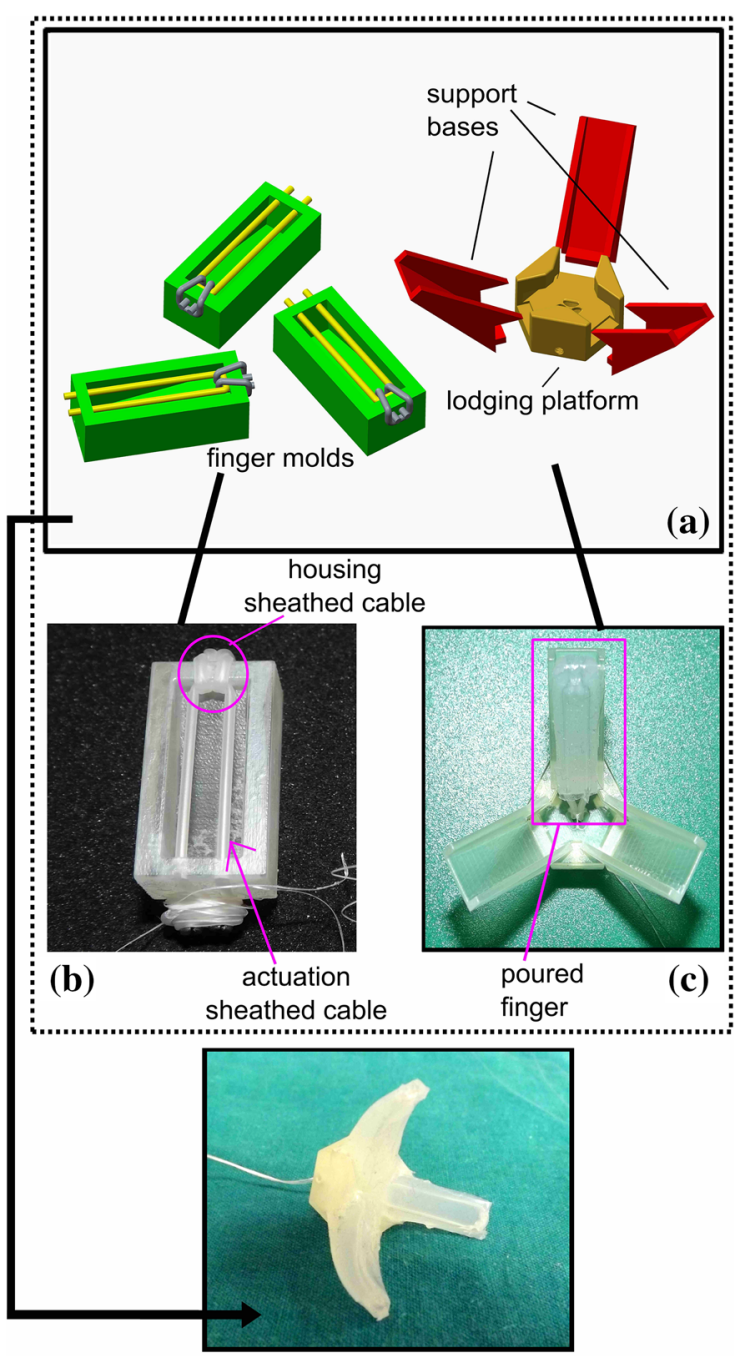

(d)

Fig. 3 a CAD design of all parts used in the casting of the gripper; finger molds shown with their respective actuation and housing cables internally arranged; b realization of cables arrangement inside the mold and $\mathbf{c}$ placing of the poured finger in the lodging platform by means of support base; $\mathbf{d}$ final silicone prototype 
are previously inserted in silicone sheaths which are necessary to allow cables to slide with reduced friction. The sheaths are made of silicone material that is properly embedded with the structure of the gripper. The housing of the actuation cable is performed using another sheathed cable fastened to the top of the mold. Both cables are put in tension and fixed around a screw (Fig. 3b);

3. The fingers are casted separately to ensure that the cables path is straight. The used polymer is a commercially available silicone (EcoFlex 00-30, Smooth-on Inc.) which consists of a Part $A$ and a Part $B$ mixed in ratio $1 A: 1 B$ (by volume or weight), and left to polymerize at ambient temperature. The mixing of the two parts of the silicone produces bubbles that are eliminated through degasing the liquid mixture in a vacuum chamber. After that, silicone is gently deposited in molds with a needleless syringe (Fig. 3c);

4. The three fingers are then placed together in the lodging platform by means of three support bases. The cables are collected and inserted into three holes in the center of the frame. Finally, a second casting phase merges the structures. Once the silicone casting is done, the prototype is ready (Fig. 3d) and the silicone sheath remains incorporated, so that the wires can slide with low friction.

In addition to standardized prototypes, there is a wide range of solutions, reported here such as examples, which can be implemented to enrich the behaviour of the gripper:

- Cable placing at different heights: different deformation behaviours can be obtained via anchorages at different heights inside the mold (Fig. 4a);

- Fingernails: in the human's hand the nails provide stability during precision grasping. A variant of gripper has been realized embedding a Delrin rectangular $\left(5 \times 6 \times 0.5 \mathrm{~mm}^{3}\right)$ fingernail in the terminal and dorsal part of it, to stiffen the fingertip (Fig. 4b and 4c);

- Grip increasing: to prevent the slippage of objects, an additional milled layer on the finger ventral surface increases the contact area and so the tribological interaction between the digital surface and the object surface. The layer is highlighted adding a red pigment to the silicone. It is obtained

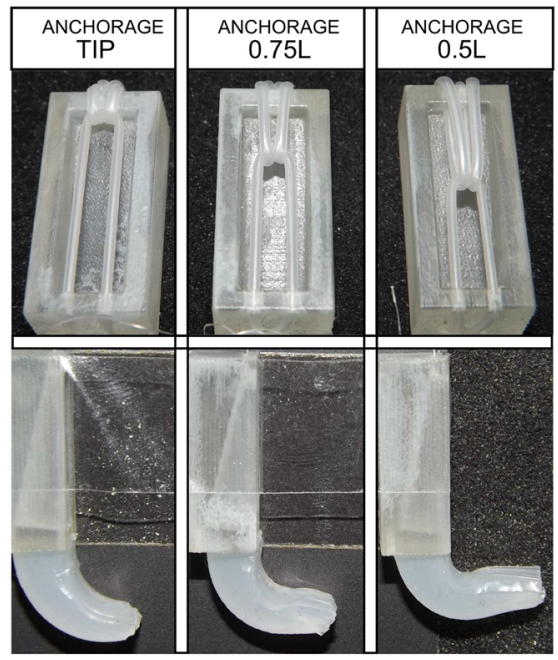

(a)

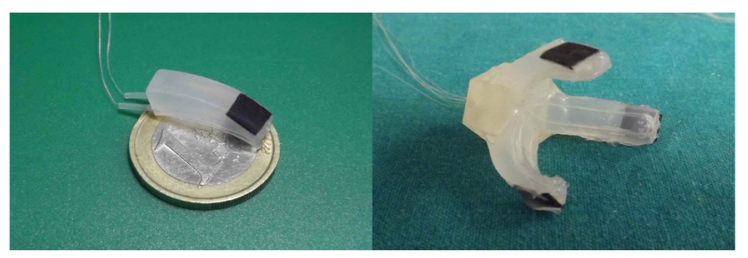

(b)

(c)

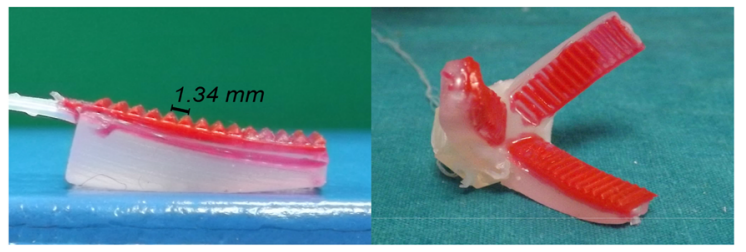

(d)

(e)

Fig. 4 a Different anchoring points of the cables from the fingers' tips and relative deformation at the same tension value; variation provided with Delrin fingernails: b finger and c corresponding gripper; variation provided with milled layer on the fingers ventral surface: $\mathbf{d}$ finger and e corresponding gripper

by casting a thin layer of silicone on a $1.34 \mathrm{~mm}$ pitch milled grid, pressing above it the ventral part of the finger and then leaving the mixture to polymerize (Fig. 4d, e).

A test bench has been specifically set up in order to perform the prototype characterization in terms of relationship between cables tension and fingertip force and for evaluating the gripper behaviour (Fig. 5a). It is 
composed of a platform, specifically an anthropomorphic robotic arm, which holds the gripper and provides its alignment to the force sensor (Fig. 5b). The whole measuring system is constrained on the platform. An anthropomorphic robotic arm has been used as the testing platform for high flexibility in the positioning of the tool and for allowing further experimental tests on grasping stability. A custom interface between the gripper and the robotic arm wrist has been designed, provided with an inner curved cavity to slide the cables, accompanying them from the frame of the gripper and bringing them out from the side, where the cables are inserted in a $2 \mathrm{~mm}$ diameter metal sheath. Real-time cables tension measurements have been carried out by means of a force gauge sensor (Alluris FMI-210B5, Freiburg, Germany) mounted on a micrometer slide system. The metal sheath incorporates the cables up to the sliding plate system, mounted on the robot elbow. Passing through a double diameter hole to block the sheath, the cables are finally fixed to the force gauge sensor interface (Fig. 5c).

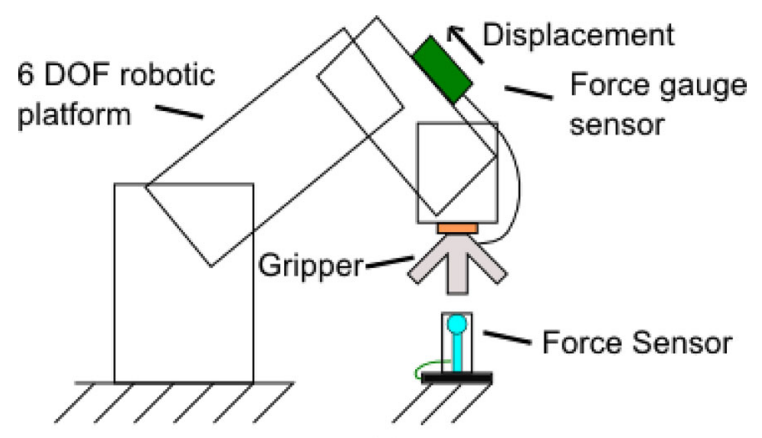

(a)

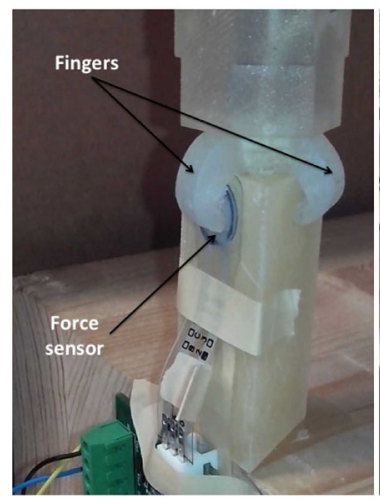

(b)

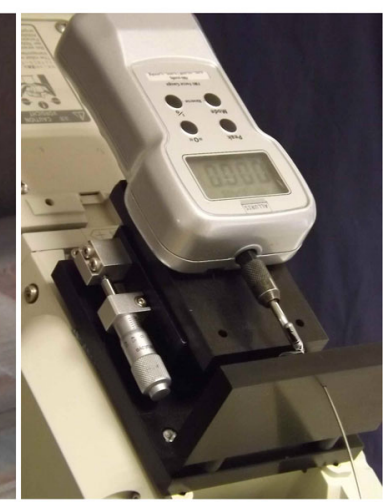

(c)
Fig. 5 Test bench: a Experimental task of fingertip force measurement, b grasping setup with prism object, force sensor and board and $\mathbf{c}$ slide system with force gauge sensor
A commercial force sensor has been used to acquire the grasping force (A201, Tekscan FlexiForce Force Sensor, South Boston, MA, USA) together with a data processing circuit (Tekscan FlexiForce QuickStart Board) connected to an Arduino Mega microcontroller board. The sensor has been previously characterized performing compression tests using a universal testing machine (Instron 4464, Torino, Italy) to derive the calibration curve. A triangular-based prism (side 25 $\mathrm{mm}$ and height $50 \mathrm{~mm}$ ) has been used as a testing object. The force sensor has been laid on one of the prism faces, with the active part facing outwards. The positioning of the object is centred in respect to the gripper and their axis are coincident. The orientation of the object is settled in order to put its face parallel to the gripper finger ventral side. Pulling the specific cable, the finger bends toward the object, up to touching the sensor active region (inserts in Fig. 6). In particular, experimental tests with 2 prototypes of the standard gripper (Fig. 3d) have been performed increasing the cable displacement by steps of $0.5 \mathrm{~mm}$ ( 5 measurements for each step) and measuring the cable tension and the force exerted by the finger itself. The test is aimed at evaluating the force behaviour (i.e., maximum force and data trend) performed by the 6 fingers belonging to the two evaluated grippers, on the same testing object, thus highlighting both intra-gripper and inter-gripper behaviours of the system.

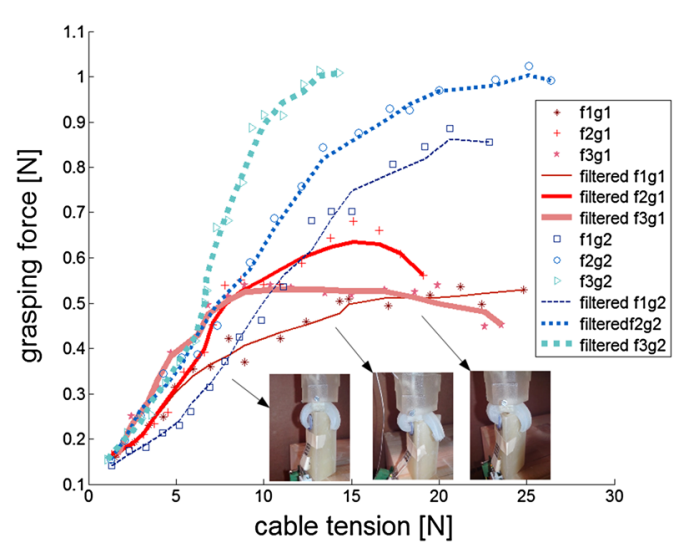

Fig. 6 Characterization results of 2 standard grippers. Experimental task of fingertip force measurement performed for each finger, one finger at the time, on gripper $1(f 1 g 1, f 2 g 1, f 3 g 1)$ and gripper $2(f 1 g 2, f 2 g 2, f 3 g 2)$. Insets represent the first finger of gripper 1 in action at three different levels of contractions. (Color figure online) 


\section{Results and discussion}

The overall results of the experiments are shown in Fig. 6. These results refer to loading cycles performed by actuating each fingers, one finger at a time, on two standard grippers. For gripper 1 the average maximum grasping force value is $0.68 \mathrm{~N}$ in correspondence of $15.12 \mathrm{~N}$ level of tension, obtained on finger 2 (medium-width solid line in Fig. 6), whereas for gripper 2 the average maximum grasping force value is $1.02 \mathrm{~N}$ in correspondence of $25.12 \mathrm{~N}$ level of tension, obtained on finger 2 (medium-width dashed line in Fig. 6). The maximum value of the cable tension has been set $50 \%$ of the maximum breaking tension (i.e. $50 \mathrm{~N}$ ); a pilot test allowed to set it, being it a reasonable value for covering the entire system behaviour. The reported results indicate that in the current version of the gripper, the system shows a similar behaviour both intra- and inter-gripper. Uncontrollable intrinsic elements in the manufacturing process led to grippers with different embedded physical behaviours and performances. Indeed, the process involves critical manual steps, such as the cables arrangement and tensioning. A fully optimization will be assessed in order to increase the repeatability of the manufacturing process. The behaviour is safe, but it is necessary to perform, at this time, a calibration of the system before its use.

The system is able to produce a maximum force which lies below the target value: for MIS it this value set around $5 \mathrm{~N}$ on the tip of the instrument during a surgical task ([5, 17], excluding retraction of organs). However, since they have the same order of magnitude (i.e. $1 \mathrm{~N}$ ), some modifications on the structural design (arrangement of the cables and geometry) or material (other elastomers with different elastic properties or composite/ anisotropic materials) could improve the robustness and allow the application of higher cable tensions. Despite some precautions included in the measuring setup, the tensioning mechanism is not optimized and presents sharp curves and not straight paths, which cause high friction and consequently energy loss.

The viscoelastic behaviour of the silicone material determined a scattering dataset of results which is clearly visible in Fig. 6. A filtering process has been applied to underline the trend of the force (moving average filter in Curve Fitting toolbox in MathWorks MATLAB environment); also the raw data (dots in the same plot) suggest that the force at the finger reaches a maximum, then it presents a sort of plateau. Plateau continues for a significant period after which the failure of the system occurs; it occurs when the load overcomes a certain threshold and the silicone body is not more able to resist to the internal stress provided by the cable. As a result, the cable rips the silicone at the tip, where the system is thinner. The explanation of this phenomenon is potentially related to the intrinsic behaviour of the finger. This structure can be assimilated to a tapered structure (thinner at the tip) that is subject to an internal pulling force. These kinds of structures tend to deform towards a tensed/contracted shape (instead of forming a more intuitive circular arc): it means that when the force overcomes a certain threshold it starts shrinking and the tip force is no more applied on the object/sensor (as represented in the inset on the right of Fig. 6); the applied force is distributed also on a tangential direction at a characteristic threshold force value on the object/sensor.

Of course part of the structure still directs its force toward the internal part of the gripper, so that while increasing the tension cable, the force applied by the finger increases accordingly, but part of this force is lost on itself and applied on a tangential direction. This result means that by optimizing the design of the fingers it may be possible to set a maximum force where the gripper is still fully functional (not damaged), but an increasing tensile force does not change the resultant gripping force on the object. The presence of the plateau assures a safe and reliable control: a maximum grasping force is reached by the system in a broad range of cable tension. In the surgical environment this is translated into the possibility for the surgeon to apply force without the necessity of a precise control and care for damages. The plateau part of the curve self-limits the stress that the gripper is able to exert and avoids tissue damages.

\section{Conclusions}

In this paper a proof of concept for a novel early stage surgical tool has been presented. It mainly exploits two basic design concepts: the use of solely soft materials and an under-actuated system which is able to adapt the finger shape to the target object and apply a certain amount of force.

The functionality has been assessed and quantitatively evaluated and the results represent a base for 
new possibilities in the use of soft bodied gripper with features which are very suitable in the medical field. They possess intrinsic safety features and self-limiting properties which guarantee an extremely safe interaction with biological tissues.

In this perspective, the impact of the lack of sensorization of surgical instruments, which still represents a limit in terms of force feedback in MIS procedures, is minimized and the designed tool, characterized by a self-regulated mechanism, paves the way for a paradigm shift in safe surgical manipulation.

Acknowledgments The authors acknowledge the contribution of N. Funaro, A. Melani, R. Lazzarini, A. Pratesi, F. Falotico, T. Mazzocchi and C. Quaglia for their help in system development.

Open Access This article is distributed under the terms of the Creative Commons Attribution 4.0 International License (http:// creativecommons.org/licenses/by/4.0/), which permits unrestricted use, distribution, and reproduction in any medium, provided you give appropriate credit to the original author(s) and the source, provide a link to the Creative Commons license, and indicate if changes were made.

\section{References}

1. Araromi OA, Gavrilovich I, Shintake J, Rosset S, Richard M, Gass V, Shea HR (2014) Rollable multisegment dielectric elastomer minimum energy structures for a deployable microsatellite gripper. Mechatron IEEE/ASME Trans 20(1):438-446

2. Bar-Cohen Y (2000) Electroactive polymers as artificial muscles-capabilities, potentials and challenges. Handb Biomim 11(8):1-3

3. Bethea BT, Okamura AM, Kitagawa M, Fitton TP, Cattaneo SM, Gott VL, Baumgartner WA, Yuh DD (2004) Application of haptic feedback to robotic surgery. J Laparoendosc Adv Surg Tech 14(3):191-195

4. Carrozza MC, Cappiello G, Micera S, Edin BB, Beccai L, Cipriani C (2006) Design of a cybernetic hand for perception and action. Biol Cybern 95(6):629-644

5. Cavusoglu MC et al (2000) Telesurgery and surgical simulation: design, modeling, and evaluation of haptic interfaces to real and virtual surgical environments. University of California, Berkeley

6. Cianchetti M, Ranzani T, Gerboni G, De Falco I, Laschi C, Menciassi A (2013) Stiff-flop surgical manipulator: mechanical design and experimental characterization of the single module. In: International conference on intelligent robots and systems (IROS), 2013 IEEE/RSJ. IEEE, pp 3576-3581

7. Deimel R, Brock O (2014) A novel type of compliant, underactuated robotic hand for dexterous grasping. In: Robotics: science and systems
8. Galvao Neto M, Ramos A, Campos J (2009) Single port laparoscopic access surgery. Tech Gastrointest Endosc 11(2):84-93

9. Gibo TL, Deo DR, Quek ZF, Okamura AM (2014) Effect of load force feedback on grip force control during teleoperation: a preliminary study. In: Haptics symposium (HAPTICS), 2014. IEEE, pp 379-383

10. Ilievski F, Mazzeo AD, Shepherd RF, Chen X, Whitesides GM (2011) Soft robotics for chemists. Angew Chem 123(8):1930-1935

11. In H, Cho K-J, Kim K, Lee B (2011) Jointless structure and under-actuation mechanism for compact hand exoskeleton. In: IEEE International Conference on rehabilitation robotics (ICORR), 2011. IEEE, pp 1-6

12. Kier WM, Stella MP (2007) The arrangement and function of octopus arm musculature and connective tissue. J Morphol 268(10):831-843

13. Kundrat D, Schoob A, Kahrs L, Ortmaier T (2014) Rapid prototyping of rod-driven continuum robots for medical applications. In: 4th Joint workshop on new technologies for computer/robot assisted surgery, pp 139-141

14. Laschi C, Cianchetti M (2014) Soft robotics: new perspectives for robot bodyware and control. Front Bioeng Biotechnol 2:3

15. Laschi C, Cianchetti M, Mazzolai B, Margheri L, Follador M, Dario P (2012) Soft robot arm inspired by the octopus. Adv Robot 26(7):709-727

16. Lirici MM (2012) Single site laparoscopic surgery: an intermediate step toward no (visible) scar surgery or the next gold standard in minimally invasive surgery? Minim Invasive Therapy Allied Technol 21(1):1-7

17. Madhani AJ (1997) Design of teleoperated surgical instruments for minimally invasive surgery. $\mathrm{PhD}$ thesis, Massachusetts Institute of Technology

18. Moradi Dalvand M, Shirinzadeh B, Nahavandi S, Smith J (2014) Effects of realistic force feedback in a robotic assisted minimally invasive surgery system. Minim Invasive Therapy Allied Technol 1-9

19. Munro MG (2002) Laparoscopic access: complications, technologies, and techniques. Curr Opin Obstet Gynecol 14(4):365-374

20. Piccigallo M, Scarfogliero U, Quaglia C, Petroni G, Valdastri P, Menciassi A, Dario P (2010) Design of a novel bimanual robotic system for single-port laparoscopy. Mechatron IEEE/ASME Trans 15(6):871-878

21. Puangmali P, Althoefer K, Seneviratne LD, Murphy D, Dasgupta P (2008) State-of-the-art in force and tactile sensing for minimally invasive surgery. Sens J IEEE 8(4):371-381

22. Quaglia C, Petroni G, Niccolini M, Caccavaro S, Dario P, Menciassi A (2014) Design of a compact robotic manipulator for single port laparoscopy. J Mech Design

23. Stassen HG, Grimbergen CA, Dankelman J (2004) Introduction to minimally invasive surgery. Eng Patient Saf Issues Minim Invasive Proced 2-18

24. Stilli A, Maghooa F, Wurdemann H, Athoefer K (2014) A new bio-inspired, antagonistically actuated and stiffness controllable manipulator. In: 4th Joint workshop on new technologies for computer/robot assisted surgery, pp 112-114

25. Suzumori K, Iikura S, Tanaka H (1991) Development of flexible microactuator and its applications to robotic 
mechanisms. In: Proceedings of IEEE International conference on robotics and automation, 1991. IEEE, pp 1622-1627

26. Tholey G, Desai JP, Castellanos AE (2005) Force feedback plays a significant role in minimally invasive surgery: results and analysis. Ann Surg 241(1):102
27. Weber B, Schneider S (2014) The effects of force feedback on surgical task performance: a meta-analytical integration. In: Haptics: neuroscience, devices, modeling, and applications. Springer, Berlin, pp 150-157 